\title{
A search for the optical counterpart to PSR B1821-24 in M 28
}

\author{
A. Golden, R. F. Butler, and A. Shearer
}

National University of Ireland, Galway, Ireland

Received 18 September 2000 / Accepted 7 March 2001

\begin{abstract}
We have analysed archival HST/WFPC2 images in both the F555W \& F814W bands of the core field of the globular cluster M 28 in an attempt to identify the optical counterpart of the magnetospherically active millisecond pulsar PSR B1821-24. Examination of the radio derived error circle yielded several potential candidates, down to a magnitude of $V \sim 24.5\left(V_{0} \sim 23.0\right)$. Each were further investigated, both in the context of the CMD of M 28, and also with regard to phenomenological models of pulsar magnetospheric emission. The latter was based on both luminosity-spindown correlations and known spectral flux density behaviour in this regime from the small population of optical pulsars observed to date. None of the potential candidates exhibited emission expected from a magnetospherically active pulsar. The fact that the magnetic field \& spin coupling for PSR B1821-24 is of a similar magnitude to that of the Crab pulsar in the vicinity of the light cylinder has suggested that the millisecond pulsar may well be an efficient nonthermal emitter. ASCA's detection of a strong synchrotron-dominated X-ray pulse fraction encourages such a viewpoint. We argue that only future dedicated 2-d high speed photometry observations of the radio error-circle can finally resolve this matter.
\end{abstract}

Key words. pulsars: general - pulsars: individual PSR B1821-24 - globular clusters: general - globular clusters: individual M 28/NGC 6626 - Techniques: image processing - Astrometry

\section{Introduction}

The confirmation by the ASCA satellite of strong hard X-ray pulsations (Saito et al. 1997) from the isolated millisecond radio pulsar PSR B1821-24 (period $\sim 3 \mathrm{~ms}$ ) in the globular cluster M 28/NGC 6626 indicated the very real possibility of detecting an optical counterpart. Despite its great age compared to the standard non-millisecond class of pulsars, and its extremely low spin derivative, the magnetic field \& spin coupling in the vicinity of the light cylinder is of a similar order to that of the younger pulsars, especially the Crab (PSR B0531+21), as was originally pointed out by Saito et al. (1997). We recall that the Pacini \& Salvati (1987) model predicts synchrotron emission occuring at some constant fraction of the light cylinder radius, and which furthermore scales with the period $P$. This theoretical framework has been shown to predict rather accurately the anticipated optical and Xray fluxes for the youngest pulsars. Indeed, by using this model, one can estimate a factor of $\sim 5$ difference between the predicted and observed X-ray luminosities for PSR B1821-24, which does seem to substantiate the initial suspicions of Saito et al. (1997) and once again validate the three decade old model of Pacini (1971), certainly in Xrays. One might argue that a definitive optical detection

Send offprint requests to: A. Golden, e-mail: agolden@itc.nuigalway.ie within the predicted range would vindicate this underlying assumption as regards the correct emission mechanism. The predicted optical luminosity via this approach is estimated at $V_{0} \sim 21.5-23.5$, which would be reduced to an observable $V \sim 23-25$ by the interstellar extinction towards M 28 of $A_{V} \sim 1.4$ mag (Davidge et al. 1996).

Previous high speed photometric studies of the core region of M 28 using photomultipliers and wide apertures of order $\sim 5^{\prime \prime}$ have limited any pulsations to $V>20.0$ (Middleditch et al. 1988). Clearly, such observations were strongly compromised by the crowded stellar vicinity: the pulsar lies $\sim 12^{\prime \prime}$ from the center of M 28. What would be required is a targeted search to $V \sim 25$ of the entire radio error circle. This would be an ideal opportunity to use a high speed 2-d photometer such as the TRIFFID instrument, which incorporates a MAMA photon counting detector. However, in order to optimise such a search, ideally one would hope to examine high resolution archival optical data in order to firstly identify the target field and secondly obtain photometry and astrometry for potential stellar targets within that field.

Previously, Sutaria (2000) performed such an analysis on a single F555W WFPC2 observation of the central region of $\mathrm{M} 28$, which yielded 8 potential optical sources within the combined radio plus HST error circle (ranging from $\left.m_{\mathrm{F} 555 \mathrm{~W}}=[18-23.7]\right)$. Any firm conclusion from this work was limited, as without a colour magnitude 
diagram (CMD) the possibility exists that any of these 8 could be foreground objects, or indeed that all of these 8 could be conventional, unevolved cluster stars. In addition, Sutaria did not place the observed results in the context of current emission theory, stating that a working upper limit for emission from the optical counterpart should be $\mathrm{m}_{\mathrm{F} 555 \mathrm{~W}} \geq 23.7$.

We thus obtained from the HST archive WFPC2 images of the central region of M 28 obtained with the F555W and F814W passbands. In the next section we outline the reduction of the various exposures in both bands, and their subsequent photometric analysis. The stars detected within the radio error circle were then isolated in the resulting CMD. Based on both expected luminosities and known optical pulsar spectral indices, we discuss the likelihood that these candidates are consistent with anticipated pulsar emission, and outline implications from this study for current and future searches for optical emission from millisecond pulsars.

\section{Data reduction and analysis}

We obtained two sets of WFPC2 images from the HST archive. The exposures were re-calibrated at the ST-ECF archive at the time of the request using the best calibration reference files and most recent software available. The first set were all taken on 12/09/97 between 12:22 and 15:59 UT in the F555W and F814W bands (11 and 12 exposures respectively). Exposure times in $\mathrm{F} 555 \mathrm{~W}$ were $2.6 \mathrm{~s}$ ( 3 images) and $140 \mathrm{~s}$ ( 8 images), and in $\mathrm{F} 814 \mathrm{~W}$ were $2.6 \mathrm{~s}$ (3 images), $160 \mathrm{~s}$ (6 images) and $180 \mathrm{~s}$ (3 images). The exposure pointings were dithered on a regular grid; we determined the dithering offsets (steps of 2.745 pixels in $X$ and $Y$ on the PC1 chip) from the world coordinate system (WCS) information in the image headers, and confirmed them by empirical measurements on the images.

The second set were all taken on on 8/08/97 between 03:34 and 05:32 UT, also in the F555W and F814W bands (4 exposures in each band). Exposure times in F555W were $4 \mathrm{~s}$ (1 image) and $140 \mathrm{~s}$ ( 3 images), and in F814W were $3 \mathrm{~s}$ (1 image) and $140 \mathrm{~s}$ ( 3 images). The exposure pointings were dithered on a regular grid; we determined and confirmed the dithering offsets (steps of 3.666 pixels in $X$ and $Y$ on the PC1 chip) in the same way as for the first set.

A full description of the data reduction techniques (which include some innovations) will be given in Butler \& Shearer (2001). Briefly, "warm" pixels in the individual exposures were fixed and "hot" pixels were flagged, using STSDAS/warmpix . Cosmic rays were flagged by an iterative process of shifting and stacking all images to a common reference position - whereby each individual exposure in turn defines that reference position. Then the exposures for each band, with their updated data quality masks, were cleaned of cosmic rays \& "unfixable" hot pixels (and other flagged pixel defects), combined (scaled and weighted by exposure time), repaired for saturation in all but the shortest exposures, subsampled by a factor of 2 , and corrected for spatial distortion, using STSDAS/drizzle.

"Drizzling" (Fruchter \& Hook 1997) is a linear reconstruction method, so such images which have already been subsampled by "drizzling" are an excellent starting point for our non-linear subsampled deconvolution approach to star detection and crowded-field photometry (Butler \& Shearer 2001). This attains a net subsampling factor of $4 \times 4$, which would improve the detection and photometry of the stars in and around the error circle corresponding to the position of PSR B1821-24.

The deconvolution-based photometry method was as follows. While the distortion corrections in STSDAS/drizzle produce a regular astrometric grid, they do not obviate the need for a spatially varying PSF; the PSF structure still varies substantially after "drizzling", due to field-dependent aberrations etc. Therefore, the first-estimate PSF model for each filter-band was produced by creating regularly "dithered" grids of Tiny Tim PSFs (Krist \& Hook 1996), then "drizzling" them in exactly the same way as the science exposures, and finally computing the spatially varying, drizzled PSF in IRAF/DAOPHOT - II. Then Maximum Entropy Method (MEM, implemented in STSDAS/mem, see e.g. Narayan \& Nityananda 1986; Skilling \& Bryan 1984) deconvolution was performed on a $6 \times 6$ grid of highly overlapping $512 \times 512$ pixel subimages of the M 28 field, each with its own subsampled PSF (appropriate to that position on the dithered frame) computed from the spatially varying model. The deconvolved subimages were then reassembled into a whole. This yielded a sharpened, subsampled image for each filter and epoch.

Significantly, the two epochs of observations were taken at different spacecraft roll angles, which meant that it was best to reduce them separately, and only register them after the subsampled deconvolution stage. This is because the interpolation errors induced by the process of registration would be greater at lower sampling, and because the combination-PSF would have a more complicated outer structure (8 diffraction spikes instead of 4 , for example). But the different image rotations lend a great advantage in eliminating deconvolution artefacts (arising from mismatched PSF structure or noise amplification) prior to determining a deep starlist: combining the 4 deconvolved images (F555W and F814W each at two epochs) with a moderate rejection threshold eliminated nearly all artefacts, because the radial structure of the PSF changes (due to the waveband dependence of the PSF shape), and furthermore the position-angle of the PSF structure on the sky changes (due to the roll angle changes). Indeed, not only are deconvolution artefacts eliminated in this way, but real features in the original/drizzled images which are often mistaken for stars (high-frequency structure in the wings of the stellar PSFs and the crossing points of diffraction spikes and diffraction rings of adjacent bright stars) are also removed prior to star detection.

This deep, sharp coadded image (shown in Fig. 1) was used for star detection with DAOPHOT-II/daofind. 


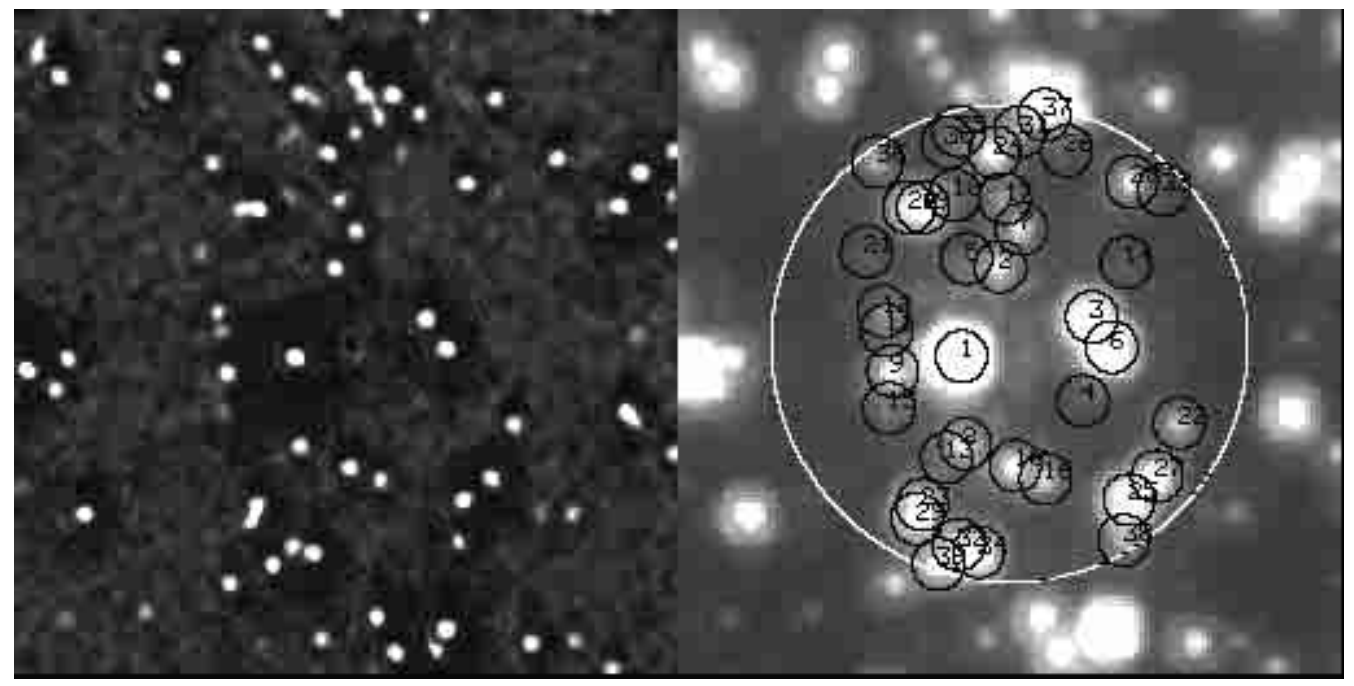

Fig. 1. Closeup of a region of the HST/WFPC2 data of M 28, 2.' 91 on a side (corresponding to 64 PC1-chip pixels), and centered on the mean nominal position of PSR B1821-24. On the left is the combined deconvolved image used for star detection; on the right is the F814W (first epoch) image after "drizzling". The adopted radio $\rightarrow$ optical error circle (radius 1.'05) containing PSR B1821-24 is shown in white on this image, and the 39 detected stars lying within this error circle are marked and numbered in black

Aperture and PSF-fitting photometry of this starlist was performed on the original "drizzled" images, and this initial photometry was used to refine the PSF models empirically; once again a subsampled, spatially varying PSF model was computed, this time using $\approx 120$ bright stars in the M 28 field. Then the subsampled MEM deconvolution steps were repeated using these refined PSF models. The final photometry was aperture photometry on these improved deconvolved images; we have shown elsewhere (Butler \& Shearer 2001) that in very crowded fields, this gives statistically better results than other methods, including the hybrid method (e.g. Yanny et al. 1994) of aperture photometry on neighbour-subtracted images. We averaged the photometry from the two epochs, after applying the correct zeropoints from Holtzman et al. (1995), correcting the photometry for charge transfer efficiency (CTE) effects using the recipe of Stetson $(1998)^{1}$, correcting for geometric distortion across the PC1 chip, correcting for the residual difference between the effective pixel sizes of the PC1 and WF3 chips after pipeline flatfielding, correcting to the "standard" 0 ". 5 radius photometry, de-extincting all measurements (adopting $E(B-V)$ of $0.41 \mathrm{mag}$ (Harris 1996) and using Table 12 of Holtzman et al. (1995) yielded $A_{\mathrm{F} 555 \mathrm{~W}}$ of $1.27 \mathrm{mag}$ and $A_{\mathrm{F} 814 \mathrm{~W}}$ of $0.77 \mathrm{mag}$ ), and finally converting from the flight $\mathrm{F} 555 \mathrm{~W}$ and F814W magnitude systems to the Johnson $V$ and Cousins $I$ bands (using Eq. (8) and Table 7 of Holtzman et al. 1995, and solving iteratively for $V_{0}$ and $I_{0}$ using the photcal package in IRAF).

We now come to the issue of astrometry; we seek to obtain the most accurate position of the pulsar in the coordinate system of our HST optical image. Two

\footnotetext{
${ }^{1}$ This of course required using the background measurements and coordinate systems of the data in their original non-deconvolved, non-drizzled form.
}

J2000 radio positions for PSR B1821-24 are quoted by Rots et al. (1998) in their Table 1: RA 18:24:32.008 Dec -24:52:11.12 (based on Jodrell Bank observations) and RA 18:24:32.008 Dec -24:52:10.70 (based on Green Bank observations). These differ by 0.42 , and although Rots et al. (1998) point out in their Sect. 4.1 that "the zero point of this [Jodrell] ephemeris is different from that of the Green Bank ephemeris" (they are almost 3 years apart), proper motion between the epoch zero points would amount to only approximately $0{ }^{\prime \prime} 01$ - far too little to account for the difference. An explanation for the discrepancy, as well as a third independent radio solution, is provided by Cognard et al. (1996). Their Nancay radio position differs from the Greenbank position by only 0 .'03 - a negligible difference compared to the uncertainty in our HST optical absolute astrometry, as we shall see below - and they quote a formal uncertainty for their position of just 0.2 mas in RA and 3.5 mas in Dec. Cognard et al. (1996) also provide the explanation for the discrepancies which can sometimes occur between reported radio pulsar positions, such as the 0.42 arcsec difference between the Jodrell and Greenbank positions in Rots et al. (1998). The explanation is the choice of ephemeris, which determines the spatial reference frame and the precise parameters of the Earth's orbital motion. According to Cognard et al. (1996), commenting on a 0.3 arcsec discrepancy, "it is expected that such large rotation exists between the reference frames of the two different ephemerides used in the analysis, namely PEP740-R for Foster \& Backer (1990) and DE202 for our solution". The more recent ephemeris is the more trustworthy one. On this basis, we proceeded with the Greenbank position, as it is in excellent agreement with the Nancay position but more recently obtained, and also taking account of another remark in Sect. 4.2 of Rots et al. (1998): "the [Green Bank] 
temporal resolution of the pulse profile is much higher than that of the Jodrell one".

However, this very accurate radio position of PSR B1821-24 maps to the HST images with a relatively large error, because of uncertainties in the HST Guide Star Catalog upon which the astrometric solution in the image headers is based. Indeed, we measured that the astrometric solutions for the two epochs differed by $1^{\prime \prime} .05$. The median absolute pointing error of HST is 0 "' 86 (Biretta et al. 2000), and the error has been measured as high as $11^{\prime \prime} 84$, while a spacecraft roll can cause an additional shift of up to 1".5 (Krist 1995). These factors explain how this same M 28 field, revisited on a different date and at a different roll angle, can have such a discrepancy in its standard image-header astrometric solution. We therefore adopted the mean predicted position of the pulsar as the centre of our search area, and examined stars out to a radius of 1.'05. The PC1 chip of the WFPC2 data comfortably encompasses this error circle. In Table 1, we list the 39 stellar point sources detected and measured within the error circle, and they are also shown overplotted on the drizzled image in Fig. 1. In Fig. 2 we overplot the CMD of the whole PC1 field with the CMD loci of all these tabulated sources.

\section{Discussion}

There is no doubt that PSR B1821-24 has an active magnetosphere, certainly in X-rays, as the observations of Saito et al. (1997) assert. In the following discussion we attempt to simply determine the general optical luminosity characteristics of the pulsar in the $V$ and $I$ bands. Thus we do not consider light curve issues etc., as what we wish to do at this stage is to test whether we have observed the optical counterpart amongst the list of 39 candidates in Table 1.

As mentioned earlier, Saito et al. (1997) pointed out that both the Crab pulsar and PSR B1821-24 share an approximately common magnetic field strength at the light cylinder. The model of Pacini \& Salvati (1987), which predicts pulsar synchrotron emission being a strong function of the light cylinder magnetic field strength. This approach has been shown to rather successfully accomodate the Xray emission from the Crab "twin" (PSR B0540-69), the Vela and PSR B1509-59 pulsars, when normalised to the emission of the Crab pulsar. As stated previously, applying Saito et al.'s (1997) observed and derived parameters of PSR B1821-24 and the Crab pulsar to this model yields a value for the predicted X-ray luminosity that differs by a factor of only $\sim 5$ from that of the observed X-ray luminosity.

The Pacini-Salvati model has also been shown to successfully accomodate the optical emission from the younger pulsars, such as PSR B0540-69 and PSR B0833-45. With imaging polarimetry at the VLT, Wagner \& Siefert (2000) have shown that the $m_{V} \sim 20$ star initially proposed as the optical counterpart to PSR B1509-59 (and subsequently rejected based on a lack of
Table 1. Photometry and astrometry of all 39 detected objects within $1^{\prime \prime}$. 05 of the mean nominal position of PSR B182124. Each drizzle pixel corresponds to 0.'0228, with the radius $R_{\text {DRIZ }}$ denoting the position of that star in relation to the mean predicted position of the pulsar, as noted in the text

\begin{tabular}{rrrrrr}
\hline ID & RA & Dec & $R_{\text {DRIZ }}$ & $V_{0}$ & $(V-I)_{0}$ \\
& $18: 24:$ & $-24: 52:$ & pixels & mag & mag \\
& ss.ssss & ss.sss & & & \\
\hline 1 & 31.9685 & 11.204 & 10.22 & 15.713 & 0.838 \\
2 & 31.9983 & 11.340 & 14.44 & 19.403 & 0.783 \\
3 & 32.0060 & 10.893 & 16.02 & 17.378 & 0.701 \\
4 & 31.9840 & 10.677 & 17.88 & 23.125 & 1.585 \\
5 & 31.9929 & 11.475 & 18.18 & 22.865 & 1.773 \\
6 & 32.0031 & 10.741 & 19.21 & 17.267 & 0.775 \\
7 & 32.0119 & 11.382 & 21.64 & 19.833 & 0.827 \\
8 & 31.9483 & 10.935 & 21.82 & 19.160 & 0.673 \\
9 & 31.9502 & 11.385 & 23.77 & 19.045 & 0.640 \\
10 & 31.9539 & 10.707 & 24.10 & 19.020 & 0.673 \\
11 & 31.9586 & 11.525 & 24.33 & 22.017 & 1.150 \\
12 & 31.9623 & 11.591 & 25.55 & 21.074 & 1.039 \\
13 & 31.9410 & 10.954 & 25.83 & 22.753 & 1.299 \\
14 & 32.0265 & 10.942 & 26.64 & 22.955 & 1.399 \\
15 & 31.9403 & 11.287 & 27.14 & 22.792 & 1.714 \\
16 & 31.9573 & 10.570 & 27.35 & 19.933 & 0.856 \\
17 & 32.0155 & 11.526 & 27.43 & 21.020 & 1.174 \\
18 & 32.0055 & 11.698 & 30.13 & 22.281 & 0.919 \\
19 & 31.9958 & 11.766 & 30.91 & 17.965 & 0.658 \\
20 & 31.9927 & 11.812 & 32.51 & 18.261 & 0.486 \\
21 & 31.9730 & 11.821 & 32.98 & 22.468 & 1.147 \\
22 & 31.9995 & 10.298 & 35.88 & 19.939 & 0.849 \\
23 & 31.9245 & 10.896 & 36.04 & 18.116 & 0.699 \\
24 & 32.0244 & 11.697 & 36.54 & 18.844 & 0.706 \\
25 & 31.9704 & 10.236 & 38.00 & 17.675 & 0.635 \\
26 & 32.0402 & 11.463 & 38.01 & 22.677 & 1.164 \\
27 & 31.9820 & 10.214 & 38.21 & 18.411 & 0.643 \\
28 & 32.0470 & 11.160 & 38.32 & 19.600 & 0.784 \\
29 & 31.9200 & 10.872 & 38.90 & 18.320 & 0.667 \\
30 & 32.0165 & 11.877 & 40.18 & 22.135 & 1.522 \\
31 & 32.0349 & 11.672 & 40.31 & 21.289 & 1.243 \\
32 & 31.9228 & 10.663 & 40.58 & 20.172 & 0.913 \\
33 & 32.0518 & 11.033 & 41.13 & 23.270 & 0.959 \\
34 & 31.9254 & 10.575 & 41.16 & 17.860 & 0.618 \\
35 & 32.0218 & 11.872 & 41.67 & 22.021 & 1.605 \\
36 & 31.9968 & 12.048 & 43.16 & 20.288 & 0.823 \\
37 & 32.0440 & 11.642 & 43.90 & 20.397 & 1.165 \\
38 & 31.9595 & 10.135 & 44.01 & 20.510 & 0.989 \\
39 & 31.9138 & 10.670 & 45.30 & 18.808 & 0.659 \\
\hline & & & & & \\
& & & & \\
& & & \\
& & & & \\
& & &
\end{tabular}

pulsations to $m_{V} \geq 23$, Shearer et al. 1998; Chakrabarty \& Kaspi 1998) actually consists of three point sources coincident with the radio position. The most strongly polarised of the three has $m_{V} \sim 25$. This is consistent with the predictions of the Pacini and Salvati model if this object is the true optical counterpart to PSR B1509-58.

A recent phenomenological study of pulsed optical emission from isolated pulsars indicates that the peak flux scales as a function of the magnetic field strength at the light cylinder (Shearer \& Golden 2001). Considering the common nature of the synchrotron emission in the optical and X-ray regime, it is reasonable to assume that the 


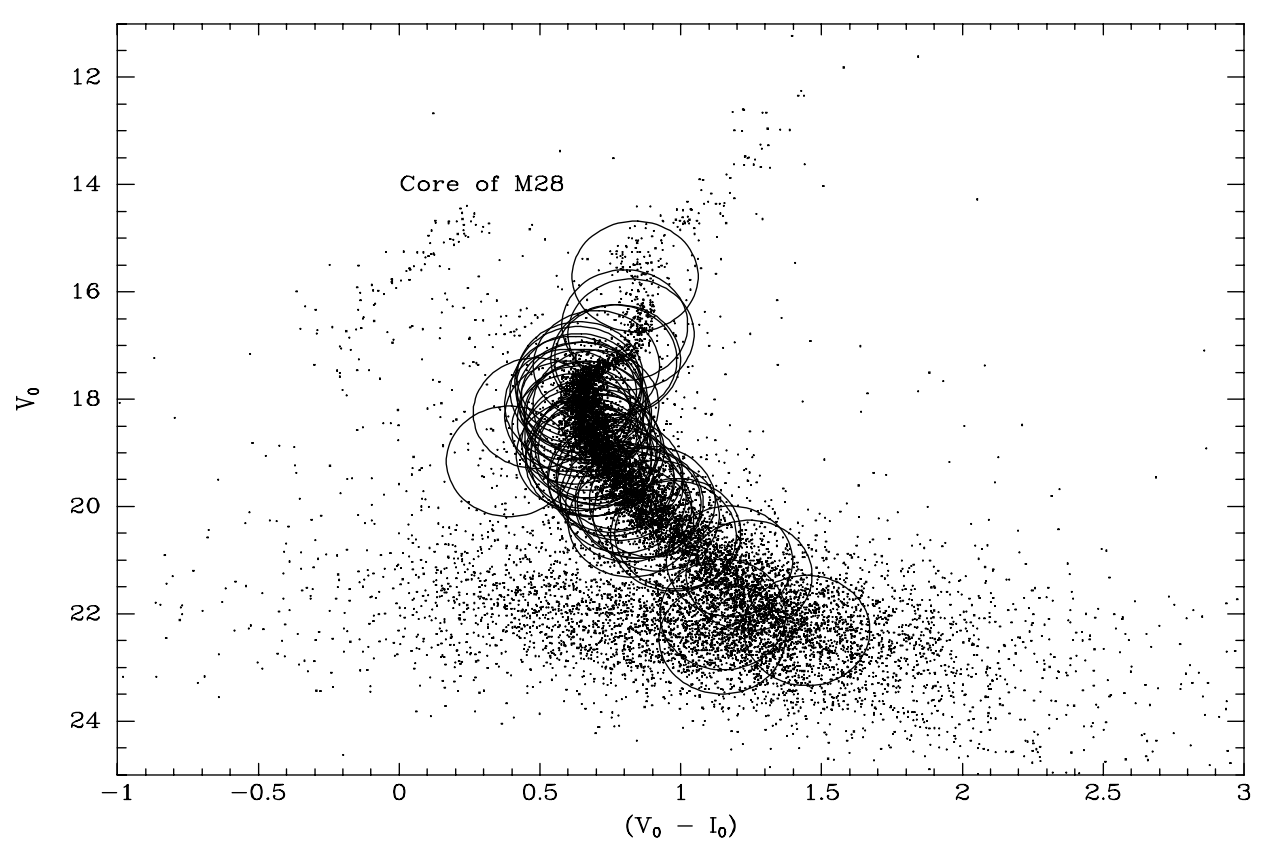

Fig. 2. CMD in $\left(V_{0}, V_{0}-I_{0}\right)$ of the HST/WFPC2 PC1-chip field centered on the core of M 28 , including the radio $\rightarrow$ optical error circle containing PSR B1821-24. Stars lying within this error circle are marked with large circular symbols. The CMD has been corrected for extinction and reddening along the line of sight to M 28

predicted optical emission from PSR B1821-28 will be a function of the light cylinder magnetic field strength, and that its predicted optical luminosity will be $V_{0} \sim$ $21.5-23.5$.

From the HST data archive, we have uncovered a number of potential candidates in our photometric analysis as listed in Table 1 . Of the 39 objects located within the combined error circle, 12 have magnitudes within that expected from the Pacini and Salvati model. The clear advantage of having $V$ and $I$ datasets means that we can test the colour index of each potential candidate to see if its emission is consistent with that of a typical main sequence star within the globular cluster core (as evident on the CMD) or whether the pronounced nonthermal emission from the pulsar results in a deviation from this. It is clear from Fig. 2 that all the 39 objects which are located within the combined error circle are consistent to first order with what one might expect from such a random sample taken from M 28, namely members of the Main Sequence and Subgiant Branch.

However definitive identification of the optical counterpart however requires an estimate of its spectral behaviour at longer wavelengths, in order to accurately predict its locus on the CMD. As there has been no clear identification of optical emission from a millisecond pulsar we must carefully consider the nature of its emission, and determine limits on the CMD within which an optical counterpart would be likely to be found. In particular we must try and determine its spectral index in the optical band.

If one naively assumes emission similar to that of the Crab pulsar, then one would anticipate an essentially flat ( $\alpha \sim 0.11$ - Percival et al. 1993; Golden et al. 2000a) power-law exponent. For a CMD corrected for the effects of interstellar extinction and reddening, this would yield a magnitude $I_{0} \geq 21.5$ and a colour $V_{0}-I_{0} \sim 0.0$ - sufficiently clear of the Main Sequence to make its identification immediate.

It is clear however that the colour on the CMD of any prospective candidate for PSR B1821-28 will be a strong function of its nonthermal spectral index, $\alpha$, particularly around $800 \mathrm{~nm}$. One possible route, in realistically trying to estimate limits for the pulsar's spectral index in the optical and X-ray regime, is to try to link the observed synchrotron relationship between the Crab with that of PSR B1821-24. Table 2 collates from the literature known spectral indices ${ }^{2}$. To first order we might expect a similar flat spectral index to that observed from the Crab pulsar.

Furthermore, based on combined thermal and nonthermal fits to observed photometry of the older pulsars PSR B0656+14 and Geminga, photon indices in the optical have been determined at $\alpha_{0656+14}=-1.4 \pm 0.6$ (Pavlov et al. 1997) and $\alpha_{\text {Geminga }}=-1.9 \pm 0.6$ (Martin et al. 1998) respectively. As both pulsars sample an evolved magnetosphere in terms of optical emission, it is useful to use these exponents as upper limits to our investigation of PSR B1821-24. Thus we predict, via the Pacini-Salvati

\footnotetext{
2 A subsequent analysis of ROSAT HRI data (Danner et al. 1997) has indicated that some fraction of PSR B1821-28's phase-averaged spectral index from ASCA may be from another X-ray source in close proximity, possibly a nebula associated with the pulsar. For the purposes of this work we assume that the spectral index from Saito et al. characterises, within error, the pulsar's emission.
} 
Table 2. Phase-averaged photon indices in the optical and $\mathrm{X}$-ray regime for the Crab-like pulsars. The X-ray data corresponds to pulse averages taken from Saito et al. (1997). The optical data for the Crab is from Golden et al. (2000a)

\begin{tabular}{ccc}
\hline Pulsar & X-ray & Optical \\
& $\alpha_{\text {x-ray }}$ & $\alpha_{\text {optical }}$ \\
\hline Crab & $-1.8 \pm 0.02$ & $0.11 \pm 0.1$ \\
PSR B1821-24 & $-1.9 \pm 0.2$ & $\sim 0.1 ?$ \\
\hline
\end{tabular}

relationship, optical pulsations at between $V_{0} \sim$ 21.5-23.5, and with a spectral index lying within the range $[0.0--2.0]$.

Thus on our CMD, this would confine the optical counterpart for PSR B1821-24 to a region defined by $21.5<V_{0}<23.5$ and $(V-I)_{0}<0$, still conspicuously to the left of the Main Sequence. It is quite clear that, to a limiting magnitude of $V_{0} \sim 23$, we have no evidence to suggest that any of the candidates are consistent with what we might expect from a magnetospherically active millisecond pulsar.

Our list of 39 objects around the nominal pulsar position should be considered as a conservative survey, as the method of preparing the summed image used for star detection deliberately removes any features which are not of similar strength in the 4 deconvolved images. This could exclude any extremely red, faint objects - i.e. those detectable in $\mathrm{F} 814 \mathrm{~W}$ but which barely register in the $\mathrm{F} 555 \mathrm{~W}$ images - but this is not a problem for this study, since, as we have explained, the pulsar is expected to have a rather flat spectrum across these two bands.

\section{Conclusion}

As yet, there have been no confirmed optical pulsations from any known millisecond pulsars. Such a detection consistent with synchrotron processes would provide crucial constraints to the nascent field of magnetospheric emission simulation, particularly those models requiring some form of "bootstrap" mechanism between the polar cap \& outer gap regions (e.g. Romani 1996). In addition, there has been considerable theoretical effort involved in determining the impact of general relativistic effects on the optical emission from millisecond pulsars (Rajagopal \& Romani 1997). Such effects are expected if the emission region is in close proximity to the neutron star surface, as would naturally be the case for thermal emission, or indeed nonthermal emission for some models. This conflicts with the evidence for apparent Pacini-Salvati scaling in the Xray regime, which argues for an emission region in close proximity to the light cylinder. Thus, detection of optical pulsations within the anticipated magnitude range would consolidate the latter model.

With this in mind, we have examined the combined HST \& radio astrometric error circle for potential optical candidates, using new image processing techniques to extend the magnitude limit of these observations in the
F555W and F814W bands and to avoid false detections. Under the assumption that the optical synchrotron emission from PSR B1821-24 follows Pacini-Salvati scaling (as is evidently the case for X-rays) and that the assumption of a common synchrotron spectral index range, we have shown that none of the potential counterparts are viable candidates. Based on this one can place the limiting magnitude for optical pulsations at $V_{0} \geq 23.0$ - which is still consistent with Pacini-Salvati scaling, and substantiates the conclusions of the preliminary study of Sutaria (2000).

It is clear that we ideally require deeper integrated photometry in order to push the limiting magnitude beyond this, and to examine any further candidates in the context of the CMD. Eventually, high speed photometry would be required to definitively identify the optical counterpart to PSR B1821-24. To perform the former would require further HST based observations, although such a prospect is unlikely given the demands on this facility. Alternatively, one could perform both deep imaging and high-speed photometry simultaneously by using a 2dimensional high speed photometer, such as the TRIFFID camera which incorporates a MAMA photon counting detector, to image the entire error circle. The present HST analysis would be used to astrometrically register the images created via binning the MAMA's 2-d time series, and all potential candidates within the error circle could then be rigorously tested for a pulsed signal using standard Fourier techniques. Indeed, the ability to choose the extraction aperture to maximise the time-series signal to noise - in contrast to earlier high speed photometry with large, fixed apertures (Middleditch et al. 1988) - provides the best opportunity to detect the pulsar. We note that previous observations using this instrument have identified pulsations from optical sources as faint as $B \sim 26.0$ (Shearer et al. 1998), as well as resolving the Crab pulsar's unpulsed component of emission (Golden et al. 2000b).

We intend to follow this archival analysis by performing such high speed observations in the near future - we base our confidence in the success of such observations on the proven ability of the TRIFFID system to detect other optical pulsars of a commensurate optical magnitude, and on the fact that there is strong evidence to indicate that PSR B1821-24 does follow the Pacini-Salvati relationship. Should such observations bear fruit, they would yield the first optical millisecond pulsar, indeed the first optical pulsar in a globular cluster or any such old stellar population.

Acknowledgements. The authors gratefully acknowledge financial support from Enterprise Ireland under the Basic Research Programme. RB also gratefully acknowledges financial support from the European Commission under the TMR programme (contract ERBFMBICT972185), under which some of this work was performed at the University of Edinburgh, UK (TMR host: Prof. Douglas Heggie). This work was based upon HST data obtained from the archive of Space Telescope - European Coordinating Facility (ST-ECF, ESO, Garching, Germany). The authors very gratefully acknowledge the assistance of the referee, Dr. Saito, in the final preparation of this manuscript. 


\section{References}

Becker, W., \& Truemper, J. 1997, A\&A, 326, 682

Biretta, J. A., et al. 2000, WFPC2 Instrument Handbook, Version 5.0 (Baltimore: STScI)

Butler, R. F., \& Shearer, A. 2001, in preparation

Chakrabarty, D., \& Kaspi, V. M. 1998, ApJ, 498, L37

Danner, R. M., Kulkarni, S. R., Saito, Y., \& Kawai, N. 1997, Nature, 388, 751

Davidge, T. J. 1996, ApJ, 468, 641

Fruchter, A., \& Hook, R. N. 1997, Proc. SPIE, 3164, 120

Golden, A., Shearer, A., Redfern, R. M., et al. 2000a, A\&A, 363,617

Golden, A., Shearer, A., \& Beskin, G. 2000b, ApJ, 535, 373

Harris, W. 1996, AJ, 112, 1487

Holtzman, J., Burrows, C., Casertano, S., et al. 1995, PASP, 107,1065

Krist, J. E. 1995, in Calibrating Hubble Space Telescope, Post Servicing Mission, ed. A. Koratkar, \& C. Leitherer (Baltimore: STScI), 311

Krist, J., \& Hook, R. 1996, Tiny Tim User's Manual, Version 4.2 (Baltimore: STScI)

Martin, C., Halpern, J. P., \& Schiminovich, D. 1998, ApJ, 494, 211

Middleditch, J. H., Imamura, J. N., \& Steiman-Cameron, T. Y. 1988, ApJ, 335, 753

Narayan, R., \& Nityananda, R. 1986, ARA\&A, 24, 127

Pacini, F. 1971, ApJ, 163, L17
Pacini, F., \& Salvati, M. 1987, ApJ, 321, 447

Pavlov, G. G., Welty, A. D., \& Cordova, F. A. 1997, ApJ, 489, 75

Percival, J. W., Biggs, J. D., Dolan, J. F., Robinson, E. L., et al. 1993, ApJ, 407, 276

Rajagopal, M., \& Romani, R. 1997, ApJ, 491, 296

Romani, R. 1996, ApJ, 470, 469

Rots, A. H., Jahoda, K., Macomb, D. J., et al. 1998, ApJ, 501, 749

Saito, Y., Kawai, N., Kamae, T., et al. 1997, ApJ, 477, L37

Shearer, A., \& Golden, A. 2001, ApJ, 547, 967

Shearer, A., Golden, A., Harfst, S., et al. 1998a, A\&A, 335, L21

Shearer, A., O'Sullivan, C. M. M., Golden, A., et al. 1998b, A\&A, 333, L16

Skilling, J., \& Bryan, R. K. 1984, MNRAS, 211, 111

Stetson, P. 1998, PASP, 110, 1448

Sutaria, F. K. 2000, in Pulsar Astronomy - 2000 and Beyond, Proceedings of the 177th Colloquium of the IAU, ed. M. Kramer, N. Wex, \& N. Wielebinski (San Francisco: ASP), 313

Wagner, S. J., \& Siefert, W. 2000, in Pulsar Astronomy 2000 and Beyond, Proceedings of the 177th Colloquium of the IAU, ed. M. Kramer, N. Wex, \& N. Wielebinski (San Francisco: ASP), 315

Yanny, B., Guhathakurta, P., Schneider, D., \& Bahcall, J. 1994, ApJ, 435, L59 